\title{
El espacio femenino en el mundo del trabajo formal
}

\author{
Iranzo, Consuelo* \\ Richter, Jacqueline**
}

\section{Resumen}

El objetivo de este artículo es presentar una panorámica de los principales problemas que confronta el personal femenino dentro del sector formal del mercado de trabajo, susceptibles de ser acometidos por el movimiento sindical a través de la negociación colectiva. El punto del cual se parte es que la invisibilidad de los asuntos atenientes al trabajo femenino, tal como la perspectiva de género ha hecho hincapié, constituye una barrera que hay que desmontar para poner en evidencia algut nas de las múltiples vías mediante las cuales se establecen diferencias de oportunidades en el mercado de trabajo. El hilo conductor del análisis es demostrar como la segregación ocupacional, la discriminación salarial, el acoso sexual, los efectos negativos de la reestructuración productiva sobre la fuerza de trabajo femenina, afectan severamente las posibilidades de inserción y de desarrollo de la mujer trabajadora dentro del mercado de trabajo. Se concluye con que el predominio de la cultura masculina en el mundo sindical, la escasa presencia de mujeres dentro de las directivas sindicales y su dificultad para traducir en reivindicaciones concretas los problemas que padecen en el mercado de trabajo, unido a la pervivencia de un cultura que coloca sobre la mujer una responsabilidad que compite a ambos sexos, son parte de los factores que hay que superar para emprender el camino que conduzca a la superación de las desigualdades actuales en materia de género.

Palabras clave: Trabajo formal, trabajo femenino, negociación colectiva, movimiento sindical.

\footnotetext{
Recibido: 02-07-27 . Aceptado: 02-09-27
}

* Socióloga del Trabajo, profesora-investigadora del Centro de Estudios del Desarrollo (CENDES) de la Universidad Central de Venezuela (UCV).

** Abogada, profesora de Sociología Jurídica de la Facultad de Ciencias Jurídicas y Políticas de la UCV e investigadora del Instituto de Derecho Privado de la misma facultad. Ambas son miembros del PPI. 


\section{Feminine Space in the Formal Work World}

\section{Abstract}

The objective of this article is to present a panorama of the principal problems confronted by female workers in the formal sector of the labor market, and susceptible to being compromised by labor unions through collective bargaining. The starting point is the invisibility of the problems of female workers, as the gender perspective activists have pointed out, which constitutes a barrier which must be eliminated in order to be able to understand the multiple ways in which opportunity differences have been established in the labor market. The central focus of the article is to point out how work segregation, salary discrimination, sexual harassment, and the negative effects of the productive reconstruction of the female labor force have severely affected insertion possibilities and the development of women workers in the labor market. The conclusion is that the predominance of the masculine culture in the labor union world, the lack of women within union directorates, and the difficulties found in translating problems found in the labor market into concrete reparations, together with the persistence of a culture which makes women uniquely responsible for what both genders must share, are all part of the problems to be overcome in order to advance along the road that leads to the overcoming of present inequalities related to gender.

Key words: Formal labor, women workers, collective bargaining, labor movement.

\section{Introducción}

La condición de la mujer en el mercado de trabajo ha sido ampliamente abordada por los estudios de género, los cuales emplean un enfoque metodológico que permite analizar las diferencias existentes entre ambos sexos en los diversos ámbitos de la vida social, revelando cómo la mujer se encuentra en franca desventaja. La perspectiva de género ha permitido demostrar como la construcción de roles y funciones en el mundo del trabajo se ha hecho básicamente tomando como patrón el modelo masculino, dejando soslayados los problemas y necesidades derivadas de la condición femenina. La división sexual del trabajo, que es una creación histórica y social determinada, llevó a la separación de la experiencia humana en dos mundos: el público de la producción (reservado al genero masculino) y, el privado de la procreación (reservado a la mujeres), lo que condujo al establecimiento de roles diferenciados y prefijados en la distribución de las diversas tareas sociales (Nelson, 1998; Bastidas, 2001). El mundo del trabajo "público y productivo" se ha construido tomando como referencia las habilidades, cualidades y necesidades de los hombres, siendo por tanto el sexo masculino la representación emblemática del obrero. En ese contexto, la mujer trabajadora se ha visto obligada a ajustarse a un patrón de comportamiento que desconoce su propia naturaleza. "Incorporar la variable género en la discusión de la división del trabajo (a través de la categoría 'división sexual del trabajo') permitió visualizar la existencia de espacios segregados dentro de las fábricas por otras lógicas que no 
son puramente económicas, y, principalmente, los mecanismos de reproducción de esa segregación" (Abramo, 1998:41).

El primer mecanismo para hacer visible las diferencias de género lo constituye la data estadística sobre el mercado de trabajo, demostrando cómo, dentro de la segmentación que lo caracteriza, una de sus principales expresiones es la representada por el espacio del trabajo femenino, en constante expansión. En América Latina, la tasa de participación femenina ha mantenido un crecimiento progresivo desde los años sesenta y entre 1990 y 1998 pasó del 39\% al 44,7\% (Abramo et al, 2000). En Venezuela particularmente, dos datos reflejan esa misma tendencia: el crecimiento sostenido de la participación femenina en la fuerza de trabajo y el aumento de su tasa de actividad. Las mujeres pasaron de ser el $31,5 \%$ de la fuerza de trabajo a principios de los noventa a constituir el $36,7 \%$ en el año $2000 \mathrm{y}$, mientras hasta 1989 su tasa de actividad no superaba el $31 \%$, para el 2001 se ubicaba en el $42,7 \%{ }^{1}$. En términos absolutos el aumento de la tasa de actividad femenina implica que en este período se incorporaron 1.619 .324 mujeres lo que equivale a un incremento de $76,3 \%$ entre estas fechas, cifra comparativamente muy superior al $34,2 \%$ de crecimiento mostrado por la oferta laboral masculina ${ }^{2}$.
Pero el aumento de la participación de la mujer en el mercado de trabajo venezolano ha venido aparejado de una disminución creciente del sector formal y un incremento del sector informal: en el año 2000 éste último generó el $52 \%$ de los puestos de trabajo y la participación femenina en su interior aumentó 12 puntos en la década -el doble de su crecimiento en la fuerza de trabajo-, mientras que el incremento de la masculina fue tan sólo del 1\% (ILDIS, 1998); para el segundo semestre del 2001 , el $52.6 \%$ de la fuerza laboral femenina se encontraba en el sector informal de la economía en comparación con el $48,2 \%$ de los hombres. En términos generales, se ha venido produciendo un decrecimiento del peso del trabajo asalariado y particularmente del femenino: en la década del noventa, la reducción del empleo asalariado fue de 8 puntos porcentuales, y para el caso del trabajo femenino la disminución alcanzó los 15 puntos porcentuales. En correspondencia, el crecimiento del empleo por cuenta propia ha sido mayor entre las mujeres que entre los hombres: en la década de los noventa, el empleo femenino en esta categoría aumentó en 16,1 puntos (pasó del 21\% en 1990 al 37\% en el año 2000), mientras que el empleo masculino se incrementó solamente 8 puntos. Estos datos nos refieren un desplazamiento de las mujeres trabajadoras hacia las formas

1 Mientras no se indique una fuente distinta, todos los datos presentados provienen de la Encuesta de Hogares por Muestreo (EHM) del Instituto Nacional de Estadística (INE), antigua OCEI. Se trabajó con la data correspondiente a los años 1990-2001.

2 El análisis de los datos entre el período 1961-90 muestra una tendencia al incremento de la participación de las mujeres casadas y unidas -las que tienen las mayores responsabilidades fami- 
de trabajo peor remuneradas de la economía no estructurada, pues, el porcentaje de mujeres no asalariadas ganado menos del salario mínimo, es 6 veces mayor que el porcentaje de los hombres (Cartaya, 1997).

Así mismo, las mujeres venezolanas han sido las más afectadas por el desempleo, tal como en el resto de la América Latina donde la brecha del desempleo entre hombres y mujeres no ha dejado de ampliarse: "mientras que en 1990 su tasa de desempleo era un $20 \%$ superior a la masculina, en 1998 esa relación se incrementa a un 47\%" (Abramo et al, 2000:12). De acuerdo a un estudio del Consejo Nacional para la Promoción de Inversiones, la brecha se profundizó en nuestro país a partir de 1992 y en el primer semestre de 2002 la tasa de desempleo femenino se ubica en $23 \%$ en comparación con el 13.6 de la masculina (Conapri, citado por Rodríguez, 2002). A la alta desocupación femenina se suma la mayor dificultad de las mujeres para encontrar empleo: según la Encuesta de Hogares por Muestreo (EHM) para el año 2000, la proporción de ellas que no había conseguido trabajo en más de un año era 16 puntos más alta que la de los hombres $(37,3 \%$ y $21,4 \%$ respectivamente).

Toda esta realidad desigual, mostrada gráficamente por la data estadística y que confirma la tendencia latinoameri- cana de feminización de la pobreza, es básicamente la consecuencia de las disímiles oportunidades ofrecidas por el mercado de trabajo para hombres y mujeres. Esta situación se ve agravada por las dificultades de las mujeres para conciliar sus actividades laborales y familiares, hechos ambos derivados de la lógica social y cultural que domina el ámbito de las relaciones sociales, marcada por las diferencias de género.

No es nuestro objetivo en este artículo tratar en extenso el tema de la mujer trabajadora, lo que exigiría, no solamente pasar revista a una complejidad de asuntos que abarcan el campo cultural, social y subjetivo, sino, además, abordar la actividad laboral femenina en sus diferentes manifestaciones como son las del trabajo doméstico ${ }^{3}$, a domicilio, por cuenta propia, como asalariada en general. Lo que aquí nos interesa es determinar aquellas áreas de problema que confronta la mujer trabajadora que debieran ser abordadas por la negociación colectiva, en tanto instrumento de regulación de las relaciones laborales, de manera de garantizar la igualdad de oportunidades para ambos sexos. Esto supone concentrarnos específicamente en el trabajo extradoméstico $y$, concretamente, en el del sector formal por ser el que está cubierto por dicha negociación colectiva. Esto no significa perder de vista las implicaciones que tiene el

liares- pasando de representar el 7 y el 5 por ciento respectivamente en 1961. al 31 y 23 por ciento en 1990, superando con ello la participación de las mujeres solteras (Zúñiga y Orlando, 2001:72,73).

3 En la literatura de género. las nociones de trabajo doméstico y extradoméstico buscan poner en evidencia la condición de trabajo de las actividades realizadas por la mujer dentro del hogar. 
trabajo doméstico para las mujeres trabajadoras, más aún cuando éstas "se incorporan al mercado de trabajo sin redefinir las responsabilidades en la esfera reproductiva" (Bethencourt, 1998:45) ${ }^{4}$, por lo que la doble jornada tiene consecuencias determinantes sobre su desenvolvimiento laboral y pasa a ser, por tanto, un asunto que concierne a la negociación colectiva. Esta es el instrumento privilegiado para que la acción consciente de los actores del sistema de relaciones de trabajo y en particular del movimiento sindical pueden atemperar e incluso revertir las lógicas segregacionistas y excluyentes del ámbito laboral.

El presente artículo tiene su origen en un estudio que hiciéramos para la Organización Internacional del Trabajo (Iranzo y Richter, 2002), como parte de una iniciativa para promover estudios comparativos sobre los avances en materia de igualdad de trato y de oportunidades en los procesos de negociación colectiva en América Latina ${ }^{5}$. Como para poder comprender los alcances y los vacíos que tienen nuestras convenciones colectivas en materia de género es necesario previamente tener claro cuáles son los asuntos a los que ésta debe hacer frente, nos pareció indispensable comenzar dando cuenta, por una parte, de los problemas que confrontan las mujeres en el trabajo y por la otra, de la situación de la mujer dentro del movimiento sindical y la postura que éste mantiene al respecto, en virtud de su importancia para velar por el cumplimiento de los derechos de todos los trabajadores/as. Y ese el objetivo de este artículo. En un trabajo posterior, abordaremos el análisis del tema de género dentro de las cláusulas de nuestra convención colectiva, pero en esta oportunidad haremos referencias puntuales al tema como hilo conductor de los retos que enfrenta el sindicalismo para mejorar la equidad y democratizar los espacios laborales.

Para situar los principales problemas que afectan a la mujer en materia laboral, en el sector formal en el caso de Venezuela, nos hemos basado en las entrevistas realizadas dentro del estudio mencionado y en una investigación que realizáramos anteriormente cuyo fin era conocer las posibilidades de inserción de las mujeres pobres en el mercado de trabajo ${ }^{6}$. La participación sindical de la mu-

4 "La reproducción de la fuerza de trabajo es un proceso complejo donde producción y reproducción forman un núcleo inseparable para contribuir a la sobrevivencia” (Bethencourt, 1998:45).

5 Proyecto regional denominado: "Negociación colectiva y equidad de género en América Latina", promovido por la Oficina Regional de la OIT para las Américas y la Oficina de Actividades para los trabajadores de la OIT. El objetivo del estudio, coordinado por Lais Abramo, es analizar la incorporación de los temas relativos a la mujer trabajadora y a las relaciones de género en el trabajo en los procesos de negociación colectiva en América Latina.

6 Este estudio "Mujeres jóvenes excluidas: un estudio cuantitativo y cualitativo" fue realizado bajo la coordinación general de Luisa Bethencourt y constó de dos partes, la primera relativa al diagnóstico de la oferta y la segunda al diagnóstico de la demanda, que es a lo que haremos referencia, y que estuvo bajo la responsabilidad de Consuelo Iranzo (Iranzo, 1998). 
jer y su escasa representación en los cargos directivos fue estudiada como una manifestación más de los famosos "techos de cristal", que ha sido el término utilizado para explicar las barreras invisibles que enfrentan las mujeres para acceder a los altos cargos gerenciales públicos y privados. Por último, no está de más resaltar que los problemas que trataremos a continuación se expresan en el segmento más privilegiado dentro del mercado laboral femenino, como es el $47 \%$ que labora en el sector formal de la economía, más aún cuando casi la mitad de éste se ubica en el sector público, que es el que goza de mayor protección.

\section{Género y trabajo formal}

Para hacer un recuento de los principales problemas que viven las mujeres trabajadoras es necesario comenzar por señalar, muy brevemente, los que la afectan en tanto reproductora de la especie y sobre quien recaen culturalmente las principales responsabilidades familiares. Esta condición tiene numerosas implicaciones directas sobre su desempeño laboral, en su mayoría harto conocidas, porque suponen un conjunto de condiciones excepcionales: en el prenatal, posibilidad de cambiar de puesto si su labor representa peligros para el feto; libertad para acudir a su control mensual, contar con ropa de trabajo adecuada a su condi- ción; seguridad de servicios de emergencia ante la contingencia de aborto; derecho a tomar su licencia antes del parto; en el postnatal, además del permiso por nacimiento, garantía de estabilidad durante el lapso de mayor dependencia del infante; derecho a amamantamiento y seguridad de un local adecuado para dejar al bebé mientras trabaja; dificultad, en virtud de sus responsabilidades familiares, para permanecer en el local de trabajo más allá de la jornada, entre los aspectos más importantes. Una gruesa parte de tales aspectos (no todos) están consagrados como derechos por parte de la legislación venezolana ${ }^{7}$, pero, a menudo, no son respetados.

El incumplimiento de la normativa laboral representa el problema más grave que sufren los/as trabajadores/as en nuestro país, inclusive dentro del sector formal, pero los estudios realizados revelan que las mujeres presentan un mayor grado de vulnerabilidad, aún en aquellos asuntos no vinculados directamente a su condición maternal. En las experiencias analizadas por Luisa Bethencourt se ponen en evidencia las numerosas infracciones a la ley que se cometen respecto al personal femenino, incluso en las empresas de más de 100 trabajadores, que suelen ser las que más la respetan: el despido anual sin pago de prestaciones, la firma de contratos en blanco, la no cancelación de los bonos que estipula ley o la

7 En nuestro artículo posterior, como marco a lo incorporado por la convención colectiva, nos referiremos concretamente a lo establecido en nuestra legislación al respecto. 
no cobertura del Seguro Social porque el patrón no paga las cotizaciones correspondientes, el pago por debajo del salario mínimo, etc. (Bethencourt, 1998) ${ }^{8}$. La frecuencia de tales infracciones aumenta a medida que disminuye el tamaño de la empresa y sobre todo en los casos en que no existe ninguna presencia sindical.

Pero más allá de los problemas derivados de su condición de reproductora y madre, las mujeres confrontan un conjunto de situaciones particulares, mucho menos visibles, que abarcan su desempeño global en el trabajo en la medida en que afectan "sus oportunidades de inserción en el mercado de trabajo y la calidad de dicha inserción" (Abramo, 1998:40), como resultado de la segregación ocupacional, la discriminación salarial y el acoso sexual de que son objeto.

\subsection{La segregación ocupacional}

El término de segregación ocupacional busca dar cuenta del relativo confinamiento que sufren segmentos de trabajadores/as a cierto tipo de puestos de trabajo, por lo general los de menor calificación, y de las restricciones con las que se enfrentan para acceder a otros por razones ajenas a sus capacidades 0 al trabajo en sí mismo. En este caso en particular, nos interesa referirnos a la segregación ocupacional femenina y aunque, como bien indica Luz Gabriela Arango, "la segregación laboral nunca es absoluta y sólo puede hablarse de tendencias y grados de segregación" (Arango, 1999:127), todos los estudios concuerdan en afirmar que ningún mercado de trabajo escapa, en mayor o menor medida, a ésta.

Como dato general de nuestro país, el $85,9 \%$ de los empleos a los que acceden las mujeres corresponde a las actividades no transables, en particular aquellas que presentan las tasas más bajas de productividad y que reportan los más bajos salarios, mientras que el empleo manufacturero femenino se ha venido reduciendo: entre 1997 y el año 2000, decreció en un 7,4\%; por lo demás, la participación femenina en la industria está mayormente concentrada en la confección, que constituye la rama con las condiciones de trabajo más desfavorables. En toda América Latina se observa el mismo acentuado proceso de terciarización del empleo femenino, pues en 1998 éste se aglomeraba en un $85,6 \%$ en el sector de servicios (cuyo $52,7 \%$ se ubica en el área con las peores condiciones, como es la de los servicios comunales, sociales y personales), mientras que la proporción del empleo masculino terciario era de un $66 \%$ (Abramo, et al, 2000:14).

La investigación en la materia ha revelado el carácter universal de la segregación ocupacional. "Estudios comparativos que intentan evaluar el impacto de la

8 A este tipo de infracciones habría que añadir las relativas específicamente a las obligaciones establecidas por vía de la legislación o de la convención colectiva respecto a las condiciones de trabajo de la mujer, las cuales las abordaremos en el próximo artículo donde analizaremos ambos instrumentos. 
industrialización sobre la segregación por sexo en realidades muy distintas, llegan a la desesperanzadora conclusión de que, a despecho de cuál haya sido el punto de partida (...) y el ritmo del proceso de industrialización, se verifica una indiscutible convergencia en la tendencia general a la segregación por sexo en el conjunto de los países analizados" (De Oliveira y Ariza, 2000:653).

Uno de los datos que hace más obvio el fenómeno de la discriminación es que la mayor participación en los puestos de baja calificación no se corresponde con los niveles educativos del personal femenino: en el caso venezolano, en el año 2000, de acuerdo a los datos de la EHM, el porcentaje de mujeres analfabetas era inferior al de los hombres $(3,5 \%$ y $5,2 \%$ respectivamente) y era mayor, en cambio, el porcentaje con formación media $(24,6 \%$ contra $19,5 \%)$ técnica $(7,5 \%$ contra $4,5 \%)$ y universitaria $(16,2 \%$ contra $9 \%$ ). Los datos sobre el empleo femenino y masculino en América Latina demuestran que las mujeres "necesitan un número de años de estudio significativamente mayor para acceder a las mismas oportunidades de empleo y de condiciones de trabajo que los hombres. En ese sentido es que se puede decir que su grado relativamente mayor de escolaridad, así como los avances verificados en la década, no garantizan a las mujeres más y mejores empleos en comparación con los hombres" (Abramo et al, 2000:16).
La segregación ocupacional tiene lugar desde el momento en que la mujer se incorpora al trabajo, pues desde el ingreso a la empresa se conforma un mercado primario, con los trabajadores con posibilidades de carrera y uno secundario constituido por los que "perciben bajos salarios, están sujetos a peores condiciones de trabajo, poseen menos beneficios sociales, los empleos son discontinuos y con pocos chances de avance dentro de la organización" (García, 1989:253).

En el estudio que hiciéramos sobre las posibilidades de inserción de las mujeres pobres en el mercado de trabajo (Iranzo, 1998) ${ }^{9}$-donde se constató que son mucho más restringidas que para los hombres-, las razones que adujeron los empresarios entrevistados para preferir mano de obra masculina en ciertos sectores o puestos, eran en su gran mayoría derivadas del mantenimiento de prácticas excluyentes fundadas en consideraciones que trascienden al trabajo en sí mismo. Si bien la restricción relativa a las condiciones físicas necesarias tiene un fundamento irrefutable en ciertos casos, en su mayoría respondía más específicamente a la imposibilidad de la mujer embarazada para realizar determinadas tareas que ponen en peligro la vida del feto, tanto en la manufactura como en el área de servicios técnicos. Al no contratar mujeres, los patronos eliminan la necesidad de realizar transferencias en caso de embarazo, con lo que crean barreras al sexo

9 A pesar de que la investigación se hizo sobre los sectores con mayor participación femenina en la industria, el comercio y los servicios, el $88 \%$ del total de los establecimientos dijo tener puestos de trabajo para los cuales prefería la contratación de hombres (Iranzo, 1998). 
femenino para acceder a puestos de trabajo para los cuales podría estar perfectamente capacitado. Otro argumento con fundamentos y consecuencias similares es la limitación que impondría la existencia de turnos rotativos en los procesos continuos en la industria o la existencia de jornadas hasta altas horas de la noche en servicios o en comercio. Aún cuando no existe en nuestro país ningún obstáculo legal para contratar mujeres en trabajos nocturnos, los empresarios dan por sentado que ellas no pueden trabajar bajo ese régimen en razón de sus obligaciones maternales; de esa manera la mujer se ve imposibilitada a decidir al respecto de manera autónoma. Fuera de estos argumentos, los restantes responden a consideraciones basadas en la costumbre, al convencimiento de la capacidad técnica inferior en la mujer o al temor a las obligaciones que les puede acarrear la embarazada y madre.

El resultado de todas estas consideraciones es que la mano de obra femenina tiene un acceso limitado a ciertos sectores y a ciertos puestos. En el caso de la industria, aparte de los procesos donde la mano de obra femenina tiene una presencia significativa (confección, cosméticos, medicamentos) ${ }^{10}$, en los restantes suele estar concentrada en las labores menos calificadas, principalmente al final de la cadena de producción (envasadora, empacadora, etiquetadora), lo que permite distinguir claramente los puestos de trabajo femenino de los masculinos (Iranzo, 1998). En el sector servicios, la participación de la mujer es muy alta en las actividades tecno-administrativas pero muy baja en las de carácter técnico-operativo (control, mantenimiento, reparación). Sin embargo, el mismo estudio nos permitió detectar algunas pocas empresas que sí contratan mujeres para todos los puestos, siendo la mejor demostración de que lo contrario es una forma de exclusión.

Otra restricción con la cual se encuentran las mujeres y que expresa también una discriminación respecto a los criterios de selección, es la situación fami$\operatorname{liar}^{11}$. Si bien en el estudio sobre mercado de trabajo (Iranzo, 1998) el 64\% de las empresas dijo no adjudicarle importancia a este aspecto, el $36 \%$ tiene como política escoger mujeres con determinada condición familiar: la mayor parte prefiere mujeres solteras sin hijos (el sector financiero y el de comercio fueron donde más importancia se le dio a esta condición), por considerar que las madres se ausentan y

10 Una rama tradicionalmente femenina como es la textil, concretamente el área de hilanderías, ha disminuido drásticamente la contratación de mujeres, habiendo sido las primeras despedidas en razón de la crisis que atraviesa el sector (entrevista Presidente del sindicato textil/confección).

11 Estudios del mercado de trabajo partiendo de la segregación por sexo, han sido sistemáticos en apuntar que ser casada o soltera, con hijos o sin hijos, edad, color, apariencia física, clase social a la que pertenece la familia, son aspectos que determinan las condiciones de inserción de la mujer en el mercado de trabajo y revelan las sensibles diferencias entre hombres y mujeres (Soares, 1998). 
retardan en demasía por la necesidad de atenderlos; en cambio, en las ramas de la industria donde mayormente se contratan mujeres, las compañías las prefieren casadas y con hijos porque juzgan que ello las hace más estables y responsables. Un $8 \%$ de las empresas entrevistadas indicó su preferencia por las mujeres que no tienen como proyecto tener hijos, asunto que, al no existir garantías, se puede vincular con la información aportada en talleres con mujeres sindicalistas, según la cual ciertas empresas están solicitando certificados de esterilización para la contratación de personal femenino (Iranzo et al, 1997).

Todas estas restricciones para acceder al empleo se ejercen apoyadas en argumentos supuestamente objetivos que a menudo son asumidos por los mismos representantes sindicales. De allí que, las condiciones de acceso de la mujer al empleo, las cuales conforman un tipo de "inserción excluída" (Posthuma, 1998:25) ${ }^{12}$, no son normalmente consideradas como un asunto que competa al sindicato o a la negociación colectiva. Es más, la práctica ilegal de exigir exámenes que garanticen la ausencia del embarazo está siendo aceptada pacíficamente por los dirigentes sindicales.

Una vez al interior de la empresa, el segundo momento que refuerza la segregación ocupacional es cuando se establecen los criterios para la formación del personal. Dentro de la poca o mediana capacitación impartida dentro de las empresas, la selección de los candidatos para los cursos (inclusive cuando hay intervención del sindicato), los horarios dentro de los que éstos son dictados y los temas seleccionados, favorecen en principio al personal masculino, fenómeno estudiado como inherente a la división sexual del trabajo. "La posición a priori desventajosa de la mujer en el mercado de trabajo, limita su acceso a las oportunidades de entrenamiento y por ende de elevar su calificación" (Phostuma, 1998: 25). En la medida en que el entrenamiento prioriza al personal masculino, se refuerzan los prejuicios y los problemas reales en torno a las capacidades técnicas de las mujeres, y se consolida un círculo vicioso sin solución aparente. Este es otro aspecto que suele ser ignorado por las instancias sindicales, aunque hay que advertir que en nuestro país el tema de la capacitación en general no suele formar parte de la negociación colectiva, por lo que es un asunto que queda en manos de la iniciativa empresarial. Entre los dirigentes sindicales entrevistados, sólo los de la empresa estatal Metro de Caracas mostraron preocupación por el tema y desde hace varios años han tenido presente el acceso equitativo a los cursos de formación (Iranzo y Richter, 2002).

La escasa capacitación de las mujeres es un importante factor para obstaculizar su movilidad horizontal y vertical, por lo que la promoción viene a represen-

12 "De acuerdo a Gove y Rodgers existen cuatro formas de exclusión social en relación al mercado de trabajo: desempleo abierto, formas precarias de inserción, formas de trabajo no remunerado y exclusión de las oportunidades para desarrollar nuevas habilidades" (Posthuma,1998:25). 
tar el tercer momento en que se refuerza la segregación ocupacional. La tendencia a mantener al personal femenino en los puestos más bajos de la escala jerárqui$\mathrm{ca}$, mediante el establecimiento de mayores trabas que al personal masculino para su ascenso profesional, independientemente de su nivel y calificación, es llamada por algunos autores como segregación vertical, para distinguirla de la tratada anteriormente, que sería la segregación horizontal (Phostuma, 1998). En las entrevistas realizadas a sindicalistas, la mayor parte reconoció que las mujeres tienen muchas más dificultades de ascender que los hombres. Excepto en los sectores mayoritariamente femeninos, son muy raras las mujeres que acceden a los puestos de dirección e incluso en aquellos, a menudo son hombres quienes ejercen las responsabilidades ejecutivas (Iranzo y Richter, 2002).

La segregación vertical ha sido estudiada con cierta profundidad en las investigaciones sobre la participación de la mujer en los cargos gerenciales como parte del fenómeno denominado "techo de cristal", esto es, las barreras artificiales e invisibles, creadas por prejuicios psicológicos y estructurales que impiden que las mujeres ocupen puestos directivos de altos rango (Wirth, 1999: 107). Los datos compilados por la Organización Internacional del trabajo (OIT) muestran avances de las mujeres en la ocupación de puestos profesionales y directivos, lo que ha sido vinculado con su mayor acceso a la educación en casi todos los países, pero la mayor presencia femenina en especialidades tradicionalmente masculinas, como la ingeniería, no se ha visto reflejada en los altos cargos gerenciales de dichas disciplinas. Wirht lo reseña claramente: "Con pocas excepciones, la proporción de mujeres que desempeñan puestos directivos rara vez excede del $2 \%$ en la mayoría de los países, a pesar de que constituyen más del $40 \%$ de la fuerza de trabajo mundial. Cuanto más alto es el puesto, más flagrante es el predominio del hombre. En las entidades mayores y más poderosas, la proporción de los altos cargos ocupados por mujeres suele oscilar entre el 2 y $3 \%$. En los Estados Unidos, donde las mujeres componen el $46 \%$ de la fuerza de trabajo, una encuesta de las 500 mayores compañías (la lista "Fortune 500") reveló que en 1996 ocupaban tan sólo el $2,4 \%$ de los puestos de alto nivel, y sumaban un diminuto $1,9 \%$ de los funcionarios y directores mejores pagados" (Wirth, 1999: 111). Similar situación ha sido reportada en varios países de América latina. En Brasil, la proporción se ubica en un 3\%. En Argentina, un estudio sobre los medios audiovisuales reveló que en los canales de televisión de un total de 170 cargos directivos sólo 13 puestos estaban ocupados por mujeres (CNM, 1999). Es importante señalar que en estos estudios también se revela que a menudo las propias mujeres no perciben la existencia de tal discriminación.

\subsection{La discriminación salarial}

La discriminación salarial es otro de los aspectos más destacados por los estudios de género, estando demostrado que existe una importante distancia entre los sueldos de la mano de obra masculina y femenina (Heide, 1999; Robison, 1998). En nuestro país, "el ingreso medio de las 
mujeres representa $76 \%$ del de los hombres para finales de la década (...) las mujeres ocupadas, para todos los niveles educativos, devengan menores remuneraciones que sus colegas hombres con niveles de escolaridad similares" (Zúñiga y Orlando, 2001:79,80). A través de la EHM se puede observar que, si bien los trabajadores del sector público perciben mejores ingresos que los del sector privado (tomando como base 100, corresponden a 129 y 97 respectivamente), las diferencias más marcadas por género se observan en el primero y particularmente entre los obreros del sector público, donde las mujeres ganan el $63,4 \%$ del salario de sus pares masculinos. La tendencia es similar en el caso de las mujeres gerentes en el sector público (que representan el $39 \%$ del total de esa categoría ocupacional), pues ellas ganan el $86 \%$ del salario de los gerentes hombres ${ }^{13}$. En el sector privado la situación de la mujer en general mejora un poco, pero hay sectores donde la situación se agrava, como en la industria textil/confección (donde el 65,2 de los ocupados son mujeres ${ }^{14}$ ), pues su salario es $36 \%$ inferior al de los hombres. Aparte de las desigualdades de salario de acuerdo al puesto, tradicionalmente femenino o masculino, otro factor, que puede llegar a tener un peso importante en la diferencia global de las remuneraciones, lo constituye las horas extras, ya que las mujeres tienen menos posibilidades de acceder a ellas. De cualquier manera, la situación en el sector formal es siempre más favorable a la del sector informal, donde la discriminación es aún más evidente: las cifras promedio indican que en éste las mujeres perciben la mitad de las remuneraciones que su mismo sexo en el sector formal.

Al igual que la segregación ocupacional, la discriminación salarial permanece como un fenómeno encubierto para la gran mayoría de los dirigentes sindicales: aquellos interrogados al respecto tendieron a negarla, pues, en su opinión, en los ámbitos donde se desenvuelven se estaría respetando uno de los principios que les es más caro: salario igual por trabajo de igual valor (Iranzo y Richter, 2002). Como la segregación ocupacional y la discriminación salarial son las dos caras de una misma moneda, en la medida en que no se percatan del primer fenómeno tampoco pueden percatarse del segundo. Como ha sido señalado por Lais Abramo, uno de los problemas a este respecto es que no existen mecanismos eficientes que permitan comparar los trabajos de igual valor (Abramo, 1998), más aún cuando los requisitos exigidos parten de ideas preconcebidas. Es decir, en la remuneración inferior en los puestos de trabajo tradicionalmente ocupados por las mujeres y con ello, en la distinción entre trabajo calificado y no calificado, está implícita una valoración social de los saberes que se consideran innatos a la mujer: por una parte, aquellos que respon-

13 Estos datos no pretenden sostener que existan diferencias de sueldo para un mismo cargo, sino demostrar como las mujeres ocupan aquellos que reciben los sueldos más bajos. 
den a un aprendizaje no formal, adquirido dentro de la dimensión doméstica, por efecto de las responsabilidades que debe asumir desde temprana edad y, por la otra, aquellos atributos más comúnmente propios al comportamiento femenino como son la minuciosidad, la habilidad manual, la disciplina, la capacidad de atención al público, que fueron los principales atributos adjudicados a la mujer por parte de los gestores de personal entrevistados (Iranzo, 1998). La facultad para sincronizar bien los movimientos, la capacidad de concentración y la resistencia a los trabajos repetitivos, son otros de los rasgos que han sido considerados más comunes en las mujeres que en los hombres (Kergoat, citada por Leite y Silva, 1997; Guilbert, 1966). La diferente ponderación de tales cualidades fue destacada desde los sesenta en un acucioso estudio sobre el trabajo femenino en la industria: "Pudimos constatar una tendencia a la valoración de una cualidad natural de la mano de obra masculina, la fuerza física, mientras que la mayor rapidez de la mano de obra femenina no interviene como un factor de calificación" (Guilbert, 1966:141).

Como bien lo han destacado los estudios sobre el tema, no son solamente los responsables de la gestión del trabajo quienes no le atribuyen a los rasgos mencionados una cualidad que se traduce en valor agregado, sino que las mismas mujeres las asumen como banales en tanto no son acreditadas, sino adquiridas por la fuerza de las circunstancias en el hogar; expresión ésta de la difusa frontera entre la esfera privada y la esfera pública en el campo de lo femenino. "El trabajo que realiza la mujer madre para generar in- gresos está, en muchos casos, tan ligado a su quehacer doméstico que no es considerado, por ellas mismas, como un 'verdadero trabajo' y por lo tanto tampoco es declarado como tal" (Bethencourt, 1998:168). "La naturaleza supuestamente corporal del trabajo que permite la naturalización de las calificaciones femeninas, su adjetivación como naturales porque inscritas en los propios cuerpos femeninos, presenta todavía, como contra cara, la negación de la adquisición de estas habilidades por las propias mujeres quienes también las naturalizan, asimilándolas a las actividades domésticas, las cuales no se configuran como trabajo, ya que están inscritas en el orden de la misma naturalización (...) Al aparecer como cuerpo, la esfera de la domesticidad y de la naturaleza es frecuentemente asociada a necesidad en oposición a libertad, configurada como acción humana" (Rizek y Leite, 1998:70,80).

La discriminación salarial dentro del análisis de la división sexual del trabajo nos revela, por tanto, una valoración de las tareas y ocupaciones que no responde a criterios de carácter técnico, sino a una lógica del poder donde los atributos masculinos están por encima de los atributos femeninos; es decir, nos remite al concepto de calificación como construcción social. Desde que Pierre Naville definiera la calificación como una relación social y como tal, dependiente de las interacciones sociales (Naville, 1956) son muchos los estudios que han cuestionado las nociones de calificación con pretensiones universalistas y que se han ocupado en develar la incidencia de mecanismos sociales en la forma de etiquetar las ocupaciones y, más concretamente, las actividades tradicio- 
nalmente femeninas (Carrillo e Iranzo, 2000; Nelson, 1998).

\subsection{El acoso sexual}

Un problema de diferente naturaleza que confronta la mujer trabajadora, pero que también podría ser objeto de políticas sindicales y regulado por la convención colectiva, es el acoso sexual.

Las entrevistas a dirigentes sindicales revelaron la existencia de este problema en varios sitios de trabajo y constituye un problema frente al cual se sienten impotentes. Por lo general, el acoso proviene de altos ejecutivos y las denuncias no han podido prosperar, siendo incluso las víctimas las que han sido despedidas. En todas las entrevistas surgieron anécdotas al respecto, pero fue especialmente llamativo que en la banca este asunto fuera reportado como uno de los más graves que se les ha presentado. Cuando se trata de supervisores, la política que suelen seguir los sindicatos es exigir la separación inmediata de las personas involucradas, asignándoles en áreas de trabajo distintas y solamente tuvimos conocimiento de una oportunidad en la que se logró despedir a un supervisor sobre quien recaían varias denuncias. Pero los mismos sindicalistas hombres reconocieron que, en el primer momento, suelen tener dudas sobre la denuncias y se preguntan si la mujer no habrá tomado la iniciativa; esto a su vez lo vinculan con la existencia de ciertas reglas informales, según las cuales el ascenso o el acceso a ciertas prebendas se les ve facilitado por la aceptación de insinuaciones de carácter sexual por parte de sus superiores. Es esa primera duda frente al origen del pro- blema la que, muy probablemente, inhibe a muchas víctimas del acoso a denunciarlo y, de hecho, todos los sindicalistas reconocieron que deben haber muchos más casos sobre los cuales no han recibido información (Iranzo y Richter, 2002).

Pero la visibilidad del problema sigue afrontando una serie de obstáculos culturales, el fuerte arraigo de la división entre lo público y lo privado en nuestro país. El tema sexual sigue siendo considerado parte de los espacios privados y como el acoso sexual afecta mayoritariamente a mujeres, las barreras para hacerlo emerger se hacen más pronunciadas, pues lo vinculado a su sexualidad está más fuertemente ligado a la esfera privada. En este aspecto pudiese estarse manifestando la cultura jurídica predominante, que si bien considera inadecuado el acoso, no lo visualiza aún como un asunto de tal gravedad como para recibir penas privativas de la libertad y tampoco se tiene plena convicción de que este hecho deba ventilarse en las instancias jurisdiccionales.

\section{Reestructuración productiva y trabajo femenino}

Los rasgos de las innovaciones de carácter técnico y organizacional producto de los procesos de reestructuración productiva, deberían, teóricamente, actuar a favor de una mejor inserción de la mujer en el trabajo, en tanto (Abramo,1996): a) con la automatización se disminuye la peligrosidad y riesgo de muchos trabajos, así como la necesidad de fuerza física para llevarlos a cabo; b) con el nuevo paradigma organizativo y la puesta en práctica de la polivalencia dis- 
minuye la separación estricta entre los puestos, integrándose tareas de diferente naturaleza, con lo que se reducen los puestos no calificados; c) ciertos rasgos atribuidos a las mujeres como la capacidad de comunicación, de cooperación, la responsabilidad, son revalorizados en la nueva cultura organizativa.

Sin embargo, diferentes estudios al respecto realizados en el sector manufacturero latinoamericano, así como el que realizáramos en el caso venezolano, coinciden en que las transformaciones tecnológicas no han modificado el papel que juega la mujer; por el contrario, en algunos aspectos lo han reforzado (Abreu, 1993; Rolfsen, 1994; Abramo, 1996; Romero y Barrón, 1998; Rizek y Leite, 1998; Iranzo, 1998: De Oliveira y Ariza, 2000) ${ }^{15}$. Los diagnósticos revelan que las mujeres continúan concentrándose en los estratos inferiores de la escala jerárquica y que la incorporación de nuevas tecnologías se ha hecho manteniendo el trabajo descalificado por parte de aquellas. De acuerdo al Instituto Nacional de Estadística y Estudios Económicos de Francia, en la década de los ochenta, al tiempo que aumentó la proporción de mujeres trabajando en líneas de montaje y de las sometidas a ritmos de trabajo impuesto, disminuyó la proporción de hombres que trabajaban en tales condiciones (Leite y Silva, 1997). Por su parte, Arango cita un impor- tante número de trabajos donde se coincide en concluir que los procesos de reorganización productiva han tenido una escasa repercusión "sobre los salarios, las calificaciones, las oportunidades de promoción y de capacitación formal de las mujeres, aún de aquellas que han desarrollado habilidades y nuevas destrezas comunicacionales" (Arango, 1999:129).

Esto ocurre no porque exista una voluntad perversa que desea mantener a las mujeres en condición de inferioridad, porque este problema no les atañe a ellas exclusivamente. Aún en las empresas industriales más modernizadas en nuestro país, no todos los trabajadores han visto enriquecer sus tareas de manera de poder hacer uso de nuevas competencias, ni todos tienen acceso a un entrenamiento que garantice su desarrollo profesional, porque ello, por lo demás, no se ajustaría a la racionalidad de los cambios en curso: ni el grado de modernización tecnológica, ni el número de puestos 'integrados', ni las formas de movilidad interna -horizontal y vertical- lo justificarían. Es decir, ni la reorganización, ni la incorporación de equipamientos automatizados, han eliminado la necesidad de trabajo manual parcelado y elemental; éste sigue operando sobre todo en la periferia de los puestos centrales del proceso, constituyendo tareas que, en la medida en que no se establece una polivalencia o movilidad

"Aún cuando el análisis de estos aspectos ha dado lugar a no pocas divergencias y puntos de desencuentro, lo cierto es que una sólida corriente de investigación ha documentado histórica y empíricamente que, salvo algunas excepciones, la tendencia secular de cambio ha seguido el camino de la masculinización a la feminización, y que éste ha estado acompañado, por regla general, del deterioro en la calidad de las ocupaciones" (De Oliveira y Ariza, 2000:654). 
general entre todos los trabajadores, pueden ser realizadas por personal de baja calificación (Iranzo, 1997).

Son tales puestos a los que siguen sujetas principalmente las mujeres, porque son los que continúan requiriendo aquellos atributos considerados tradicionalmente como femeninos, mientras que, en los puestos centrales, las capacidades técnicas y las competencias intelectuales pasan a ser más valoradas que las manuales. En las empresas estudiadas nos encontramos con una relación negativa entre las políticas modernizadoras y las políticas frente a la mano de obra femenina: en diversas fábricas nos informaron que, cuando modernizan su maquinaria e incorporan equipos computarizados, en vez de reentrenar a las trabajadoras manuales, las sustituyen por hombres, a quienes consideran mejor preparados para manejarlas (Iranzo, 1998). Es decir, están feminizando en forma más evidente las categorías de operarios no calificados. Lo mismo fue observado en un estudio sobre el trabajo fabril en Sao Paulo, donde el cambio tecnológico trajo consigo un proceso significativo de masculinización, sobre todo en los puestos en que se produjo una integración entre las tareas de operación y mantenimiento, y una mayor segregación de las mujeres a los puestos más taylorizados (simples y repetitivos) (Rizeck y Leite, 1998).

El paradigma que envuelve a la reestructuración productiva es el de la flexibilización y uno de sus pilares fundamentales es el de la flexibilización del trabajo, cuya caras más extendidas es la de su flexibilización externa; es decir, la externalización de los costos laborales por medio de la subcontratación. Aunque éste no es un fenómeno nuevo, ha adquirido con la globalización una renovada fuerza y sofisticación. La forma más antigua de subcontratación es el trabajo a domicilio, eminentemente femenino, que en los hechos está fuera del resguardo del movimiento sindical, pero a éste se suman otras formas igualmente precarias en tanto exentas de toda protección social, como son el trabajo a tiempo parcial o determinado (en sus diferentes modalidades) y el trabajo subcontratado a través de otras empresas. La flexibilización del trabajo externa se ha convertido en la forma por excelencia de abaratamiento del trabajo, pero ella tampoco escapa a la segregación ocupacional, pues las mujeres son las primeras candidatas a ocupar los puestos flexibilizados, en correspondencia con el tipo de tareas que les son asignadas y que son de más fácil externalización; esto nos fue corroborado en las entrevistas a los sindicalistas, particularmente de los sectores de la salud, textil/confección y calzado. Este fenómeno ha sido destacado por la literatura sobre la materia: "en años recientes ha surgido la inquietud acerca de la medida en que los procesos de flexibilización de las relaciones laborales han estado asociados con una creciente precarización de las condiciones de trabajo y de feminización de la mano de obra" (De Oliveira y Ariza, 2000:648).

La incidencia particular de la intensificación del trabajo precario sobre las mujeres no es un fenómeno exclusivo de América Latina: "Según datos del Mercado Común europeo, el $70 \%$ de los nuevos puestos de trabajo creados entre 1985 y 1992, considerados descalificados se encuentran en el sector servicios, en tiempo 
parcial y ocupados por mujeres" (Arango, 1999:126). De allí que en un estudio del caso francés se afirme que la palabra flexibilización remite a realidades muy diferentes para el sexo masculino y para el femenino: el primero tiende a identificarla con reprofesionalización, el segundo con precarización (Arango, 1999). Así mismo, los estudios sobre cadenas productivas han puesto de relieve que las redes de subcontratación, que se generan como parte del proceso de desintegración vertical, descansan en importante medida en el uso de trabajo precario y uno de sus pilares es el trabajo femenino (Castillo y Santos, 1998). Incluso en el caso de los distritos industriales del norte de Italia, catalogada como una experiencia virtuosa de desagregación productiva, se ha observado una pronunciada división sexual del trabajo, al punto que "el trabajo femenino cumple un importante papel en la garantía de la flexibilidad" (Leite, citada por Abramo, 1998:46).

La crisis económica en la cual se han visto envueltos la mayor parte de los países de América Latina ha profundizado los efectos negativos de la reestructuración económica sobre el empleo y sobre las condiciones de trabajo. El influjo de la tesis, muy discutida, de que la fuerza de trabajo femenina es más costosa que la masculina, las suele convertir en las primeras víctimas de las medidas de reducción de plantillas ${ }^{16}$. En Venezuela, después de los jóvenes, las mujeres son las que tienen las tasas más altas de desempleo y ello a pesar de que un estudio reciente concluyó que el costo de emplearlas es tan sólo 1,9\% superior al de los hombres (Conapri, citado por Rodríguez, 2002). Un empresario y director de un estudio de opinión daba una razón que a menudo hemos escuchado a los empresarios y que puede ser aún de mayor peso: "en época de recesión los riesgos de maternidad convierten a las mujeres en un personal inflexible, que no puede ser despedido por gozar del período postnatal" (Luis Vicente León, citado por Rodríguez, 2002).

Sin embargo, así como la maternidad y su régimen de protección pudiese incidir negativamente sobre las posibilidades de empleo de la mujer, hay otros aspectos que pudiesen actuar a su favor en momentos de crisis. En primer lugar, los sectores más afectados en las dos últimas décadas han sido los de la industria más masculinizada. "Si bien la segregación sexual del mercado de trabajo reduce las opciones para las mujeres, puede actuar de modo indirecto como factor de 'protección' relativa en la medida en que han sido precisamente ciertas ocupaciones femeninas típicas (sectores asalariados de la manufactura y el terciario) las menos impactadas por la crisis, mientras las actividades masculinas en las industrias intermedias y de capital

“...la mano de obra masculina no internaliza las externalidades negativas producidas por el tener y cuidar una familia y un hogar, y al mismo tiempo no tiene que remunerar los servicios que obtiene a partir de la división sexual del trabajo todavía vigente (...) Y es esta desigual distribución de los costos de la reproducción social lo que contribuye a explicar, más allá de lo que pue 
han sido las más afectadas" (De Oliveira y Ariza, 2000:647). En segundo lugar, la facilidad de externallización del trabajo femenino mencionada anteriormente, junto con el costo inferior que supone el tipo de tareas subcontratadas y en particular el trabajo doméstico, pudiese también actuar como aliciente para ser uso de la mano de obra femenina que ha superado la edad reproductiva (Idem). De cualquier manera, estos factores no parecen ser lo suficientemente importantes como para impedir que la tasa global de crecimiento del desempleo femenino sea mayor que la del masculino, posiblemente por la incidencia, no tan sólo del temor a los costos, directos e indirectos, de la maternidad, sino además, de la fuerte y larga crisis de sectores feminizados como el de la confección.

La combinación de las diferentes prácticas de la reestructuración productiva han terminado por conformar una realidad laboral que en su conjunto es muy distinta a la del pasado, que era mucho más homogénea; ahora conviven trabajadores con gran diversidad de calificaciones, contenidos de trabajo, ingresos, formas de contratación y con posibilidades disímiles de movilidad dentro del mercado de trabajo (Iranzo y Patruyo, 2001). De allí que en la reconceptualización del trabajo debe estar implícita esa nueva heterogeneidad y una de sus más importantes vertientes es la constituida por las diferencias de género. "Las prácti- cas, la conciencia, las representaciones, las condiciones de trabajo y de desempleo de los trabajadores y de las trabajadoras son casi siempre asimétricas, y por tanto, razonar en términos de unidad de la clase trabajadora sin considerar el sexo social lleva a un conocimiento equivocado -o peor falso- de lo que es una clase social" (Hirata y Kergoat, citado por Delgado, 1995:150).

La nueva realidad laboral complejiza enormemente la acción sindical por la heterogeneidad de intereses que debe representar y hacer cristalizar dentro de la convención colectiva. La factibilidad de que esta última se convierta en un instrumento de combate de las desigualdades en el mundo de trabajo depende de que los propios afectados tomen parte dentro de las negociaciones, pero, concretamente en el caso de las mujeres, ese es otro espacio cuyo acceso ha estado restringido, pues el tema de la discriminación no escapa al mismo mundo sindical.

\section{Lo femenino en el mundo sindical}

La participación sindical de la mujer venezolana en los sindicatos es superior a su participación en la fuerza de trabajo. En el periodo 1994-1998, las mujeres eran, en promedio, el $36 \%$ de la fuerza de trabajo asalariada y los hombres el $64 \%$; en cambio, en el mismo periodo, las mujeres representaban el $45 \%$ de los sindi-

dan decir los datos, la persistencia de la impresión de costo relativo más alto para las mujeres que para los varones" (Lerda y Todaro:1998:310). 
calizados y los hombres el $55 \%{ }^{17}$. Incluso en un sector como la construcción, por esencia masculino, la tasa de sindicación femenina es superior a la de los hombres. La tasa general de sindicalización ha venido decreciendo desde mediados de los ochenta y ello se pronunció en los noventa (entre 1994 y 1998 disminuyó en 8 puntos porcentuales), pero la tendencia ha sido a una mayor disminución de la afiliación masculina: entre 1994 y 1998 la tasa de afiliación masculina cayó en un 16,9\% mientras que la femenina solamente en un $7,2 \%{ }^{18}$. Todo esto sitúa la tasa de sindicalización femenina venezolana por encima de los promedios mundiales y ello se explica fundamentalmente por las características de la inserción laboral femenina en nuestro mercado de trabajo, predominantemente dependiente y con un $23 \%$ en el sector público, que es el que concentra la mayor proporción de trabajadores sindicalizados. Pero su concentración en este sector no explica por si sola la tasa de sindicación femenina. La explicación que nos resulta más convincente nos la proporcionó una dirigente sindical entrevistada (Iranzo y Richter,
2002): "La mujer tiende a sindicalizarse más que el hombre porque ella entiende que el sindicato le sirve para muchos fines, que tienen que ver con la propia salvaguarda de sus condiciones de trabajo y por supuesto de la familia" ${ }^{19}$. Pareciera que la mujer tiene conciencia de su mayor vulnerabilidad en el trabajo, en especial durante los embarazos y primeros años de vida de sus hijos, y de allí que visualice al sindicato como una instancia de protección. Adicionalmente, muchos beneficios socioeconómicos son más fáciles de obtener con la mediación del sindicato, como es el caso de la caja de ahorro, los descuentos en ciertos bienes y la obtención de otros para los hijos, tales como los juguetes de fin de año, los útiles escolares. Es decir, la valoración de la protección y la mayor facilidad en la obtención de ciertos beneficios sociales, muy vinculados a la familia, se presentan como importantes alicientes para la incorporación de la mujer al sindicato.

Pero, el hecho de que las tasas de sindicalización femenina sean relativamente altas, no quiere decir que las mujeres tengan una sensible participación en

17 Los datos sobre afiliación sindical con los que contamos se limitan al período 1994-1998, debido a que fue durante esos años que la OCEI (actual INE) incorporó en la Encuesta de Hogares por Muestreo una sección destinada a explorar la situación de la seguridad social y dentro de ésta, lo relativo a la afiliación sindical.

18 La caída global ha sido muy fuerte: entre 1988 y 1998 el número de trabajadores sindicalizados disminuyó en un $26,6 \%$; al tiempo que las tasas de sindicalización caían dramáticamente al pasar de 40,3\% en 1988 a 23,9\% en 1998 .

19 Esperanza Hermida, presidenta del sindicato tribunalicio (agosto, 2001). Esta dirigente también señaló: "La mujer es como más echada pa'lante en el sector tribunalicio Asume la cuestión sindical, pero lamentablemente no podemos hablar en términos estáticos. En el tiempo de conflictos laborales las mujeres se incorporan muchísimo, pero en épocas de paz, los problemas personales, las limitaciones impuestas por los hijos, el colegio, el trabajo, etc., hacen que la mujer baje el perfil de la participación sindical. Es oscilante". 
la dirección de las organizaciones sindicales; por el contrario, ésta es muy baja. En un trabajo sobre la participación de las mujeres en los sindicatos del área metropolitana de Caracas, realizado en el año 1993, se indicaba que sólo el 19,9\% de los miembros de las juntas directivas de los sindicatos de base eran mujeres (Balliache y Febres, 1993). Esta tendencia no se ha revertido y los sindicatos siguen siendo dirigidos por hombres, incluso los que afilian mayoritariamente mujeres. En el año 1999, se reformó el Reglamento Electoral Nacional de la Confederación de Trabajadores de Venezuela (CTV), estableciéndose en su artículo 10 que "por lo menos, un treinta (30) por ciento de las postulaciones para cargos en la juntas directivas o comités ejecutivos de las organizaciones sindicales y gremiales afiliadas, deberán ser ocupadas por mujeres trabajadoras". No obstante, en las elecciones efectuadas en los sindicatos de base durante el año 2001 esta obligación no parece haberse cumplido y los datos que se manejan reflejan poca presencia femenina en su conducción ${ }^{20}$. Así mismo, en la actual composición de las directivas sindicales federadas y confederadas, la presencia de la mujer es muy baja: de las 23 federaciones regionales, 4 están presididas por mujeres y de los 17 puestos del Comité Ejecutivo, sólo 2 correspondieron a mujeres.
A manera de ejemplo y de acuerdo a las entrevistas realizadas (Iranzo y Richter, 2002): no hay ninguna mujer en la directiva del sindicato siderúrgico, sector predominantemente masculino, pero tampoco la hay en uno de los principales sindicatos del sector farmacéutico (donde la participación femenina es mayoritaria) ni en el sindicato de telecomunicaciones de Caracas (donde la mujer representa la mitad de los empleados). La relación es de una mujer por cada tres hombres en las juntas directivas de los sindicatos: del sector financiero (donde la afiliación de las mujeres es mayoritaria), de la prensa y del metro (donde la mitad de la fuerza de trabajo son mujeres), de textil/confección, (donde si bien la presencia femenina es muy alta en confección, alrededor del $80 \%$, en textil es hoy en día muy baja); y del calzado (donde la mujer representa aproximadamente el $30 \%$ de la fuerza de trabajo). En el sector de la salud (donde mayoritariamente son mujeres), la participación femenina dentro de la junta directiva del sindicato de Caracas es de un $50 \%$, pero los cinco primeros cargos están ocupados por hombres y no hay ninguna mujer en la federación correspondiente. La mayor participación femenina se da en la junta directiva del sindicato de los trabajadores tribunalicios, donde la presidenta y la mayoría de sus integrantes son mujeres, lo que en este

20 En el año 2001 se efectuaron más de 2000 elecciones sindicales de base, organizadas por el Consejo Nacional Electoral. Lamentablemente no se ha podido acceder a esa información, pues dicho organismo sólo ha entregado resultados parciales y no ha publicado una memoria oficial sobre dicho proceso. La baja presencia femenina en las directivas sindicales explica por qué la mayor parte de nuestras entrevistas fueron con sindicalistas hombres, quienes son los que adelantan la negociación colectiva (Iranzo y Richter, 2002). 
caso si se corresponde con su alta participación en el sector. En las funciones donde, por lo general, aumenta la participación femenina son las representadas por las delegaturas por sindicato 0 departamento, las cuales se dedican fundamentalmente a solucionar problemas cotidianos del sitio de trabajo y tienen poca injerencia en la toma de decisiones sobre la política sindical.

Para los sindicalistas del sexo masculino entrevistados (Iranzo y Richter, 2002), la baja participación femenina en los cargos sindicales responde principalmente a sus responsabilidades como madre, pero también al miedo escénico y a la baja formación en materia sindical, lo que explicaría que aún cuando haya mujeres sentadas en la mesa de negociaciones, su participación sea ínfima: "están como invitados de piedra" lo calificó el presidente del sindicato textil/confección; "la voz cantante la llevan los hombres", indicó el presidente del sindicato de la salud quien también señaló que las mujeres tienden a preferir representantes hombres ${ }^{21}$ (Iranzo y Richter, 2002). Es decir, constatan un hecho pero no se sienten corresponsables de la causa que lo origina, las cuales competirían básicamente a las mismas mujeres. No hay, por tanto, conciencia de que "la mayoría numérica de los hombres implica prácticas, comportamientos, lenguaje, símbolos, que excluyen a las mujeres, además de la estructu- ra de funcionamiento de las entidades y la organización del cotidiano sindical, adversos a la participación de la gran mayoría de ellas" (Delgado, 1995:152). Esto nos lo confirmó la actual presidenta de FetraAragua, para quien no necesariamente la ausencia de dirigentes bien formadas explica la poca participación de la mujer en las direcciones sindicales, sino que ellas deben enfrentar los prejuicios de sus propios compañeros. En su caso tuvo que luchar muy fuertemente dentro de su corriente sindical para presidir su plancha y uno de los argumentos esgrimidos por su contrincante interno era justamente su condición de mujer. En telecomunicaciones, las dirigentes sindicales informaron de las fuertes resistencias que tuvieron que enfrentar para reformar los estatutos en coherencia con lo establecido en el mencionado Reglamento Electoral de la CTV relativo al porcentaje de participación femenina en las planchas (Iranzo y Richter, 2002).

La escasa participación sindical femenina en las directivas es un problema general de América Latina. "Con frecuencia, las trabajadoras están subrepresentadas en la dirigencia sindical, incluso en empresas y ramas productivas en las cuales responden por un porcentaje significativo, cuando no mayoritario, de la fuerza de trabajo. En muchos casos se observa la falta de receptividad de los sindicatos para modificar una cultura esen-

21 En este sindicato, en unas elecciones de hace unos años se presentó una plancha compuesta mayoritariamente por mujeres y no ganó, siendo el electorado mayoritariamente femenino. El dirigente señaló que el nivel de formación de las mujeres era muy bueno, pero no lograron convencer a sus bases, "justamente por ser mujeres". 
cialmente masculina, que no reconoce rango sindical a muchos problemas propios del género y a veces se resiste a la incorporación de mujeres en los niveles directivos. Por otra parte, las dificultades de tiempo de las propias trabajadoras, que tienen que combinar sus responsabilidades laborales y familiares, son barreras adicionales a la participación femenina en la vida sindical" (Abramo et al, 2000:60). En Brasil, si bien en términos generales los problemas son similares, han avanzado más en estas materias que el resto de los países latinoamericanos: en la década de los ochenta, los movimientos feministas ejercieron presiones que favorecieron, junto con las propias transformaciones del mundo sindical (desarrollo y consolidación de la CUT), la constitución de comisiones y departamentos femeninos en los sindicatos que permitieron a las mujeres discutir sus realidades específicas y darlas a conocer en el medio sindical (Delgado, 1995). Por nuestra parte, no logramos conocer ningún sindicato que tuviese tales comisiones $y$, si bien existen a nivel de las confederaciones, tienen el carácter de departamentos de "la mujer y el menor", lo que de por sí refleja la concepción que atraviesa la creación del departamento, pues pone a las mujeres a cargo de la problemática de los niños y de la familia. Además con ello se asumen en igualdad de condiciones los problemas de un incapaz jurídico, como lo es el niño trabajador, con las difi- cultades que enfrenta la mujer. El departamento de la mujer de la CTV no se ha caracterizado por desarrollar o impulsar políticas de genero y tampoco ha privilegiado sus relaciones con las organizaciones feministas.

En el diseño de la política sindical, las pocas mujeres que participan no presentan temas o problemas diversos a los planteados por los dirigentes varones. El tema de la estabilidad laboral y la pérdida de beneficios socioeconómicos son las dos prioridades en el discurso sindical, independiente del sexo del dirigente ${ }^{22}$. De manera que las mujeres, o no están presentes en la mesa de negociaciones, o si lo están no se atreven o no saben argumentar, o por temor a ser discriminadas asumen las mismas prioridades que los sindicalistas hombres. La dificultad para darle carácter prioritario a los asuntos laborales femeninos puede tener también que ver conque "las mujeres trabajadoras parecen no lograr traducir sus necesidades y deseos en reivindicaciones concretas, susceptibles de ser materializadas mediante la negociación colectiva" (Soares, 1998:153).

\section{A modo de conclusión}

Sobre la base de todo lo planteado y complementando un acucioso estudio brasileño sobre los diversos problemas que afectan a ambos sexos en el mercado de trabajo (CUT et al, 1999), sintetizamos

22 Si bien esto es lógico desde los noventa, dada la situación recesiva que ha venido atravesando el país, la tendencia a concentrarse en los problemas salariales es una característica propia de la negociación colectiva en Venezuela. 
a continuación los problemas más relevantes que suelen confrontar el personal femenino para ingresar y desarrollarse profesionalmente dentro del trabajo, los cuales debieran formar parte de las preocupaciones de la negociación colectiva:

- Desatención a las condiciones de la maternidad/paternidad: a) trabas para conceder los permisos para el control prenatal; b) resistencia a transferir a las mujeres en caso de embarazo; c) ausencia de provisión de la vestimenta adecuada; d) dificuttades para el reingreso de las mujeres después de la licencia por maternidad; e) falta de local para amamantar y/o resistencia a conceder los tiempos necesarios; f) falta de guarderías o incumplimiento de los pagos correspondientes; g) ausencia de protección a padres y madres adoptantes; h) resistencia a conceder permisos para atender hijos o familiares enfermos; i) inexistencia de ayudas por parte de la empresa para la familia del trabajador.

- Mal tratamiento de los temas de salud ocupacional: a) desconocimiento sobre la salud ocupacional y su relación con la salud reproductiva de hombres y mujeres; b) desatención de las enfermedades típicamente femeninas; c) desconocimiento de los problemas producidos por los períodos menstruales y falta de provisión de toallas higiénicas; d) alta discrecionalidad empresarial para decidir los riegos que pueden afectar a las trabajadoras.

- Preconceptos en relación a la contratación y en el acceso a las carreras: a) dificultades de ingreso de las mujeres en puestos o carreras considera- das masculinas; b) mayores exigencias a las mujeres que a los hombres para el acceso a puestos de trabajo, c) preferencia por mujeres en los puestos más precarios.

- Discriminación en la formación profesional: a) poco acceso de las mujeres a los cursos de formación profesional y a los programas de calificación y reentrenamiento de las empresas; b) programas de formación fuera del horario de trabajo; c) restricción de la formación a las mujeres principalmente en áreas consideradas femeninas.

- Mecanismos de promoción y de evaluación del desempeño "sexistas": a) disminución de las posibilidades de ascenso profesional de las mujeres; b) mayores exigencias de desempeño en el caso de las mujeres; exigencia de disponibilidad fuera de la jornada de trabajo; c) exigencia de movilidad geográfica.

- Condiciones salariales que desfavorecen a las mujeres: a) diferencias salariales entre puestos "masculinos" $y$ "femeninos"; b) desvalorización de las habilidades consideradas femeninas; c) fuerte peso de las horas extras dentro de las remuneraciones; d) menosprecio de las propias mujeres acerca de sus atributos.

- Acoso sexual: a) presión física y sicológica sobre mujeres u hombres: a) exigencia de concesión de favores sexuales para preservar el empleo; b) prejuicios para encarar los problemas de acoso sexual.

- Flexibilización del trabajo femenino: a) exclusión de las mujeres en los programas de nuevas tecnologías; 
b) permanencia de las mujeres en los puestos de trabajo más atrasados; c) externalización de los puestos femeninos; c) mayor riesgo para las mujeres de ser despedidas en los momentos de crisis; d) utilización de la mano de obra femenina como variable de ajuste de los costos de las empresas.

- Discriminación dentro del mundo sindical: a) cultura y prácticas machistas en el ejercicio sindical; b) escasa presencia femenina en las directivas sindicales; c) ausencia de prioridad de los problemas de la mujer trabajadora; d) dificultad de las mujeres para plantear sus propias reivindicaciones.

En la actualidad no existe todavía real conciencia sobre la situación de la mujer en el trabajo, ni sobre las causas que la originan. En la medida en que la construcción de una sociedad más equitativa y democrática pasa por desmontar las relaciones de poder de carácter desigual, es indispensable confrontar las iniquidades resultantes de la división sexual del trabajo como una de sus expresiones. Por ello es tan importante cuestionar los roles que tradicionalmente se le asignan a hombres y mujeres, pues los mismos sustentan procesos de concentración del poder, de dominio y apropiación de unos seres humanos por otros (Bastidas, 2000: 27). A largo plazo, la resolución definitiva al problema pasa por un cambio radical en la forma de concebir la vinculación ente la vida familiar y la vida profesional, para que llegue a ser entendida como un asunto que concierne a lo femenino tanto como a lo masculino "Esta discusión está relacionada con el Estado, con las empresas, con las condiciones que se ofrecen para que las mujeres puedan ingresar en el mundo masculino del trabajo $y$ los hombres ingresen en el espacio femenino de lo social y de lo privado" (Soares, 1998:166).

Para que ello tenga lugar, es indispensable que el movimiento sindical trascienda su visión actual, comenzando por comprender todos los problemas que están envueltos en la problemática del trabajo femenino. El sindicato, como es un espacio político con lazos muy estrechos con el sistema político, responde fuertemente a patrones masculinos de ejercicio de poder. La ausencia de una perspectiva de género abarca tanto la reflexión como la acción de los sindicatos. El asunto ha sido incorporado a nivel discursivo por algunos diregentes nacionales, pero sin una concreción en la actuación cotidiana y menos como preocupación que deba proyectarse hacia las bases. La atención al tema de la mujer por parte del sindicalismo ha estado más bien restringida a garantizar el cumplimiento de los derechos legales que le confiere a ésta su condición de madre, desatendiendo los otros aspectos que rodean la situación laboral femenina, vinculados o no a su condición reproductora, pero que en todo caso la trascienden. "Las trabajadoras se tornan visibles para el sindicalismo (aunque no sea ni tanto) cuando se destaca su condición reproductora. Pero no son sujeto de política sindical cuando se trata de la dimensión 'masculina' del trabajo" (Delgado, 1995:152).

En la baja participación femenina tal vez incida también el hecho de que visualizan al sindicato como una instancia más política que gremial, y de allí que prefieren incorporarse a través de las delegaturas, 
vinculadas más directamente con la cotidianidad en el sitio de trabajo. No es casual que aquellas mujeres que han llegado a las dirigencias sindicales tengan o hayan tenido una activa militancia política partida y que su actuación sindical forme parte de un proyecto político partidista de más largo alcance, cosa que no siempre ocurre en el caso de los hombres.

La importancia actual de los derechos de la mujer en el trabajo se manifiesta en el lugar que ha comenzado a ocupar a nivel internacional, formando parte de las prioridades que se ha establecido la OIT dentro de su acción centrada en el establecimiento de un "trabajo decente", ya que éste pasa por garantizar la igualdad de trato y oportunidades entre los sexos (Roger, 2000). De allí el énfasis y la importancia trascendental que se le está adjudicando al hecho de que todos los problemas mencionados figuren como parte de la agenda sindical, en vistas a ser traducidos en reivindicaciones concretas que puedan ser incorporadas dentro de los pliegos de peticiones que se discuten en los procesos de negociación colectiva.

\section{Bibliografía Citada}

Abramo, Lais (1996), "Sociología do trabalo na America Latina: novos paradigmas produtivos relacoes de trabalho e relacoes de gênero" en Carlos Alá Santiago y Elsa Plana Larrinaga: Reestructuración productiva, cambio tecnológico, género y sindicalismo en América Latina. Ed. ALAST, San Juan.

Abramo, Lais (1998), "Um loar de genero. Visibilizando precarizacoes ao longo das cadeias produtivas" en Lais Abramo y
Alicia Rangel: Genero e trabalho na sociología latino-americana. Ed. ALAST/SERT, Sao Paulo.

Abramo, Lais; Valenzuela, María Elena y Pollack, Molly (2000), Equidad de género en el mundo del trabajo en América Latina. Avances y desafíos cinco años después de Beijing. OIT, $n^{\circ}$ 130, Lima.

Abreu, Alicia (1993), "Mudanca tecnológica e genero no Brasil: primeras reflexoes" en Novos Estudos, CEBRAP, $N^{\circ} 35$, marzo, Rio de Janeiro.

Arango, Luz Gabriela (1999), "Género, globalización y restructuración productiva" en Luz Gabriela Aranfo y Carmen Marina López: Globalización, apertura económica y relaciones indsutriales en América Latina. Facultad de Ciencias Humanas UN, Bogotá.

Balliache, Dilcia y Febres, Carlos Eduardo (1993), Participación de la mujer en los sindicatos en Venezuela. ULA/ILDIS, Caracas.

Bastidas Aliga, María (2001), Participación laboral y sindical de las mujeres en el Perú durante los noventa. Oficina Internacional del Trabajo. Oficina de Actividades para los Trabajadores. AECl. Perú.

Bethencourt, Luisa (1998), Mujeres, trabajo y vida cotidiana. Ed. CENDES, Caracas.

Carrillo, Jorge e Iranzo, Consuelo (2000), "Calificación y competencias laborales en América Latina" en Enrique de la Garza Toledo: Tratado Latinoamericano de Sociología del Trabajo. Ed. El Colegio de México/FALCSO/UAM/ Fondo de Cultura Económica, México.

Cartaya, Vanessa (coord.) (1997), Venezuela: Exclusión e integración ¿Una síntesis en construcción? Instituto Internacional de Estudios Laborales. OIT, Ginebra. 
Castillo, Juan José y Santos, Maxi (1998), "La cualificación del trabajo y los distritos industriales" en A la búsqueda del trabajo perdido, Ed. Tecnos, Madrid.

Consejo Nacional de la Mujer de Argentina (C.N.M), (1999), Memoria Anual, Buenos Aires.

DIESSE (1999), Mapa das questoes de gênero. CUT, CGT, Forza Sindical y DIESSE, Sao Paulo.

De Oliveira, Orlandina y Ariza, Marina (2000), Trabajo femenino en América Latina: un recuento de los principales enfoques analíticos" en Enrique de la Garza Toledo: Tratado Latinoamericano de Sociología del Trabajo. Ed. El Colegio de México/FALCSO/UAM/ Fondo de Cultura Económica, México.

Delgado, Maria Berenice Godinho (1995), Sindicalismo latino-americano: el difícil diálogo entre mulheres e homens" en María Silva Portella de Castro y Achim Wachendorfer: Sindicalismo Latinoamericano: entre la renovación y la resignación. ILDIS/Nueva Sociedad, Caracas.

García Fanelli, Ana María (1998), "Patrones de desigualdad social en la sociedad moderna: una revisión de la literatura sobre ocupación y salarios por género" en Desarrollo Económico, V. 29, No114.

Guilbert, Madeleine (1966), Les fonctions des femmes dans l'industrie. Ed. Mouton, Paris.

HEIDE, Ingeborg (1999), “ Medidas Supranacionales contra la Discriminación Sexual. Igualdad Salarial y de Trato en la Unión Europea" en Revista Internacional del Trabajo, número 4. Ginebra.

ILDIS (1998), Informe Social 31997 Venezuela. Ed. ILDIS, Caracas.

Iranzo, Consuelo (1997), "Gestión de la mano de obra y nuevas competencias en los sectores siderúrgicos y automotriz ve- nezolanos" en $\mathrm{M}^{\mathrm{a}}$ Antonia Gallart (edit): Competitividad, redes productivas y competencias laborales. Ed. OIT/CINTERFOR /Red Latinoamericana de Educación y Trabajo.

Iranzo, Consuelo (1998), Diagnóstico de la demanda de personal femenino en el mercado formal de trabajo" en Luisa Bethencourt: Mujeres jóvenes excluidas: un estudio cuantitativo $y$ cualitativo. Informe final de investigación. CENDES/ Ministerio de la Familia, Caracas.

Iranzo, Consuelo (coord), Bethencourt, Luisa, Lucena, Héctor y Sandoval, Fausto (1997), Relaciones laborales al desnudo. Catálogo de equivocaciones en la competitividad venezolana, Ed. Venezuela Competitiva/ CENDES, Caracas.

Iranzo, Consuelo y Patruyo, Thanali (2001), "Consecuencias de la reestructuración económica y política en el sindicalismo venezolano" en el Dossier: El sindicalismo latinoamericano en el marco de la globalización: crisis y retos a comienzos de siglo. Revista Cuadernos del CENDES, Año 18, segunda época, mayo-agosto, Caracas.

Iranzo, Consuelo y Richter, Jacqueline (2002), "Negociación colectiva y género. Estudio del caso venezolano". Informe final de investigación. Caracas, mimeo.

Leite, Marcia de Paula y Silva, Roque Aparecido (1997), "La Sociología del trabajo frente a la reestructuración productiva: una discusión teórica" en Sociología del Trabajo, nueva época, $\mathrm{N}^{\circ} 25$, otoño, Madrid.

Lerda, Sandra y Todaro, Rosalba (1998), "¿Cuánto cuestan las mujeres? Un análisis de costos laborales por sexo" en Lais Abramo y Alicia Rangel: Genero e trabalho na sociología latino-americana. Ed. ALAST/SERT, Sao Paulo. 
Naville, Pierre (1956), "Essai sur le calification du travail. Ed. Riviere, Paris.

Nelson, Julie (1998), “Trabajo, Sexo y División entre lo Económico y lo Social" en Revista Internacional del Trabajo, número 1, Ginebra.

Póstuma, Anne Caroline (1998), "Mercado de trabalho e exclusao social da forca de trábalo femenina" en Lais Abramo y Alicia Rangel: Genero e trabalho na sociología latino-americana. Ed. ALAST/SERT, Sao Paulo.

Rizek, Cibela S. y Leite, Marcia de Paula (1998), "Dimenoes e representacoes do trábalo fabril feminino" en Lais Abramo y Alicia Rangel: Genero e trabalho na sociología latino-americana. Ed. ALAST/SERT, Sao Paulo.

Robinson, Derek (1998), " Diferencias de Remuneración entre los Sexos" en Revista Internacional del Trabajo, número 1, Ginebra.

Rogers, Janine (2000), "Avances hacia la igualdad de género, pero "parciales e irregulares"' en Revista Internacional del Trabajo, número 1, Ginebra.

Romero, Mónica y Barrón, Claudia (1998), "Nuevas tecnología y mujeres jóvenes" en Mujeres trabajadoras, México (mimeo).
Rodriguez Pons, Corina (2002), "Los jóvenes profesionales y las mujeres son los primeros en las estadísticas de desempleo" en El Nacional, 14/07/2002, Cuerpo D, pág. 1.

Rolfsen, Liliana (1994), "A insercao da mulher no proceso de difusao da automacao bancaria: quesotes para debate" en Leda Gitahy: Reestructuración productiva, trabajo y educación en América Latina. CID/CINTERFOR/ UNESCO, Campinas,SP.

Soares, Vera (1998), "Acordos coletivos e as trabalhadoras" en Lais Abramo y Alicia Rangel: Genero e trabalho na sociología latino-americana. Ed. ALAST/SERT, Sao Paulo.

Wirth, Linda (1999), "Las mujeres en puestos de dirección: ¿Más cerca de romper las barreras invisibles" en Perspectivas. Revista Internacional del Trabajo, número 1, Ginebra.

Zúñiga, Genny y Orlando, María Beatriz (2001), "Trabajo femenino y brecha de ingresos por género en Venezuela" en Papeles de Población, nueva época, año $7, \mathrm{~N}^{\circ} 27$, enero-marzo, Ciudad de México. 